\title{
Medial insufficiency in postoperative weight-bearing radiographs in supination-external rotation type 4 bimalleolar ankle fractures: is the Lauge-Hansen classification insufficient in predicting medial soft tissue damage?
}

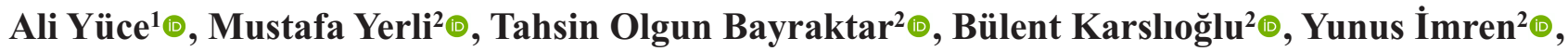

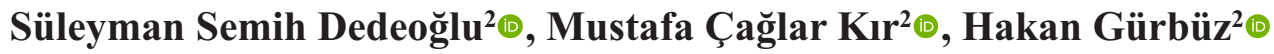

${ }^{I}$ Department of Orthopedics and Traumatology, Başakşehir Pine and Sakura City Hospital, Istanbul, Turkey

${ }^{2}$ Department of Orthopedics and Traumatology, Prof. Dr. Cemil Taşcloğlu City Hospital, Istanbul, Turkey

\begin{abstract}
Objectives: According to the Lauge-Hansen classification, supination external-rotation (SER) type 4 fractures should be accompanied by medial malleolar fracture or deltoid ligament injury. The aim of the study was to investigate medial insufficiency rates in postoperative weight-bearing radiographs in SER type 4 bimalleolar fractures.

Methods: The files of the patients who were operated with the diagnosis of SER type 4 bimalleolar ankle fracture between 2017-2020 were evaluated retrospectively. Thirty-seven cases (15 males and 22 females) were included in the study. The data based on the evaluation of the weight-bearing radiographs of the patients taken in the postoperative 1st year were examined statistically.

Results: The injury mechanism was sports injury in $17(45.9 \%)$ cases, traffic accidents in $8(21.6 \%)$ cases, falling in $9(24.3 \%)$ cases, and falling from height in $3(8.2 \%)$ cases. The preoperative tibiofibular distance was $6.05 \pm 1.86 \mathrm{~mm}$, and the postoperative tibiofibular distance was $4.19 \pm 0.40 \mathrm{~mm}(p=0.001)$. The preoperative tibiofibular overlap was $5.03 \pm 2.93 \mathrm{~mm}$, and the postoperative tibiofibular overlap was $8.62 \pm$ $1.04 \mathrm{~mm}(p=0.001)$. The postoperative medial clear space was $4.11 \pm 0.57 \mathrm{~mm}$. Postoperative medial clear space of $5 \mathrm{~mm}$ and higher was determined in 7 (18.9\%) cases.

Conclusions: In SER type 4 bimalleolar fractures with a large medial malleolar fragment fracture, weightbearing radiographs may show an increase in medial clear space. This means that a medial malleolar fracture in bimalleolar fractures may be also accompanied by deltoid ligament injury. The Lauge-Hansen classification system may be insufficient to identify a medial ligament injury.
\end{abstract}

Keywords: bimalleol, fracture, supination-external rotation, Lauge-Hansen, deltoid ligament

$\mathrm{T}$ The Lauge-Hansen classification system is often used for ankle fractures. Supination-external rotation (SER) injury is the most common type among all ankle fractures (accounting for up to 85\%) [1]. In
SER type 4 fractures, a lateral malleolar fracture is accompanied by either a medial malleolar fracture or a deltoid ligament rupture, which are treated surgically $[2,3]$. 
The purpose of ankle fracture treatment is to correct tibiotalar joint congruity and stability; thus, identifying ligament injuries in the formation of an ankle fracture is critical for treatment decisions [4]. In the literature, there are findings suggesting that in SER type 4 bimalleolar fractures, a medial malleolar fracture may be accompanied by a deltoid ligament injury [5-8]. However, these studies reported this either as a MRI finding or associated it with small fragment fractures at the collicular level at which the deltoid ligament is attached [5-8].

The Lauge-Hansen classification may not provide sufficient information to predict the fracture mechanism and soft tissue injuries $[5,9]$. We suggest that SER type 4 bimalleolar fractures according to the Lauge-Hansen classification as well as large fragment medial malleolar fractures in the supracollicular region may be concomitantly accompanied by deltoid ligament insufficiency depending on the mechanism and transferred energy. Thus, we aimed to investigate the presence of cases with increased medial clear space in weight-bearing mortise radiographs taken at postoperative $1^{\text {st }}$ year among the cases with SER type 4 injury who were operated with the diagnosis of a bimalleolar fracture.

\section{METHODS}

The files of the patients who were operated with the diagnosis of ankle fracture between 2017-2020 were evaluated retrospectively. The preoperative computed tomography (CT) images were examined, and only the images of the cases with bimalleolar fracture were evaluated. Based on the CT images, the cases (lateral + medial) were categorized by two surgeons according to the Lauge-Hansen classification. In the case of disagreement between two observers, a decision was made after examination of the images by two more persons [10]. Pediatric cases, cases with pilon fractures, cases with an additional injury, cases with a neuropathic disease, cases with neurovascular injury, cases with a pathologic fracture, cases with other type of rotational ankle fracture, cases with concomitant posterior malleolar fracture (including avulsion fracture), cases who undergone two-stage surgical protocol with external fixator, cases with postoperative tibiofibular joint incongruence, cases with insufficient postoperative reduction, and cases with a small medial malleolar fragment fracture (at collicular level) were not included in the study. The files of 37 patients diagnosed with supination-external rotational type 4 bimalleolar fracture (lateral+medial) based on the Lauge-Hansen classification were included in the study.

All the cases were operated in supine position under spinal anesthesia. In all the cases, the fracture was fixed with both lateral and medial skin incision. For stabilization of the lateral malleolus, posterolateral anatomic distal fibula plate (by TST - Turkish Spinal Trauma company) was used. The medial malleolar fracture was fixed using 2 malleolar screws or tension band technique. In the cases having syndesmotic insufficiency determined in external rotation test after the fracture stabilization, the syndesmotic screw was passed through the appropriate hole on the plate (at least 3 cortex) to provide syndesmotic stability. Then, the operation was ended by checking the stability of the syndesmosis with external rotation testing. A hemovac drain was placed in all the cases, which was removed within 48 hours after the operation. All the cases were immobilized in a short leg splint for 7-14 days, which then was removed to allow the joint movements. They were allowed for non-weight bearing walking for up to 6 weeks after the operation; then they were allowed for weight-bearing as tolerated. The weight-bearing was increased over time, and full weight-bearing was allowed according to radiography or clinical union findings.

The postoperative direct radiography and fluoroscopy images were evaluated based on the criteria of anteroposterior tibiofibular overlap (TFO) (normal $>6 \mathrm{~mm}$ ), tibiofibular clear space $(\mathrm{TCS})$ (normal $<6$ $\mathrm{mm}$ ) and medial clear space (MCS) (normal $<5 \mathrm{~mm}$ ). The cases with values outside normal values for these criteria were considered as having tibiofibular incongruence [11]. In the evaluation of the intraoperative syndesmotic joint, the same criteria were used. The postoperative radiographic measurements were based on weight-bearing radiographs.

The monitoring software, INFINITT PACS (Picture Archiving and Communication Systems) version 3.0.11.4(BN13), which was available in our hospital, was used for the pre-operative CT and post-operative radiographic measurements. The postoperative measurements were evaluated based on the radiographs 
taken in the $1^{\text {st }}$ year after operation. The measurements were performed 2 surgeons who did not perform the operations, and then they were averaged. The numerical data obtained based on these measurements were used as data in statistical calculations.

The cases whose the fracture line was located on both colliculus based on the preoperative computed tomography images were included in the study. The cases whose fracture line was at or below colliculus level (e.g.: small fragment oblique fractures) were not included in the study (Fig. 1).

The cases were operated by 3 different surgeons. All the surgeons had more than 4 years of experience in trauma surgery. The total number of lateral malleolar fracture operations performed by them in the last 3 years was more than 15 per year.

The information of the patients such as age, sex, fractured side, injury mechanism, fracture type according to the Lauge-hansen classification, lateral malleolar fracture type according to the Denis-weber classification, the presence of preoperative syndesmosis dysfunction, the use of a syndesmotic screw, fracture union times, preoperative and postoperative TFO values, preoperative and postoperative TCS values and postoperative MCS values were recorded and used in the statistical analysis.

\section{Statistical Analysis}

The conformity of the data with normal distribution was tested with the Shaphiro wilk test, the two independent group comparisons of parameters with normal distribution was examined with the Student $t$ test, and the two independent group comparisons of parameters without normal distribution was examined with the Mann Whitney U test. The relationship structure of categorical data was analyzed with the Fisher Exact and Pearson Chi-Square tests. As a descriptive statistic, mean \pm standard deviation values were given for the numeric variables, while number and \% values were given for the categorical variables. The statistical analyses were performed using SPSS Windows version 23.0 package program, and a result with $p<0.05$ was considered to be statistically significant.

\section{RESULTS}

Fifteen (40.5\%) males and $22(59.5 \%)$ females patients were included in the study. The average age was $49.30 \pm 13.50$ years. The fracture affected the right extremity in $20(54.1 \%)$ cases and the left extremity in $17(45.9 \%)$ cases. The injury mechanism was sports injury in $17(45.9 \%)$ cases, traffic accidents in 8
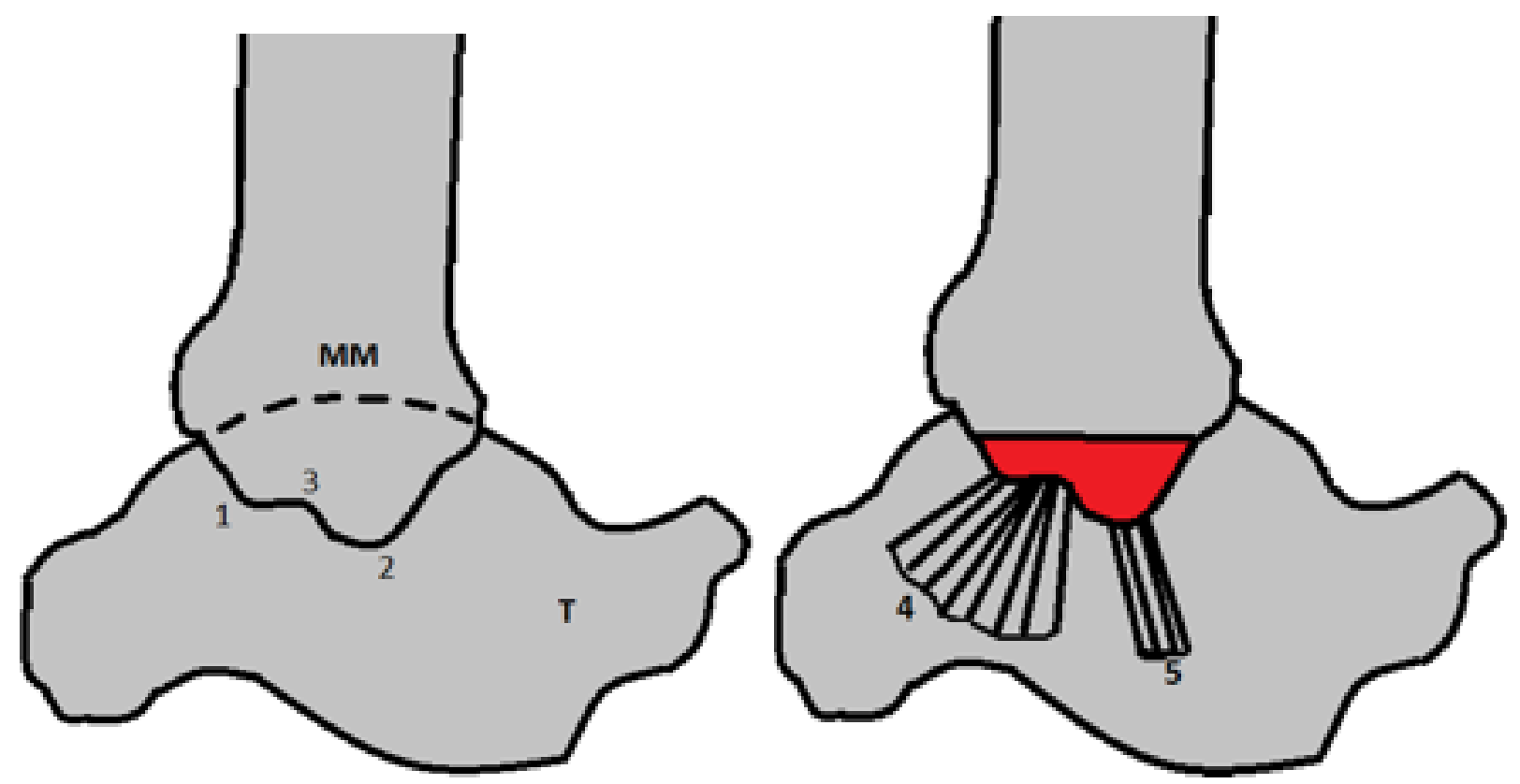

Fig. 1. The fracture line in the medial malleolus was examined by the preoperative CT images. The fractures involving the region associated with anterior and posterior colliculus (including red region) were not included in the study. (1-posterior colliculus, 2- anterior colliculus, 3-intercollicular groove, 4-deep posterior tibiotalar ligament, 5-deep anterior tibiotalar ligament, $\mathbf{T}=$ talus, $\mathrm{MM}=$ medial malleolus). 


\begin{tabular}{|c|c|c|c|c|c|}
\hline & \multicolumn{2}{|c|}{ Preoperative } & \multicolumn{2}{|c|}{ Postoperative } & \multirow[b]{2}{*}{ p value } \\
\hline & Mean \pm SD & M [Q1-Q3] & Mean \pm SD & M [Q1-Q3] & \\
\hline TCS & $6.05 \pm 1.85$ & $6[5-6]$ & $4.19 \pm 1.85$ & 4 [4-4] & $<0.001$ \\
\hline TFO & $5.03 \pm 2.93$ & $6[3-7]$ & $8.62 \pm 1.04$ & 9 [8-9] & $<0.001$ \\
\hline
\end{tabular}

$\mathrm{M}=$ median, $\mathrm{Q}=$ Quartile, $\mathrm{SD}=$ standard deviation, $\mathrm{TFO}=$ tibiofibular overlap, $\mathrm{TCS}=$ tibiofibular clear space

$(21.6 \%)$ cases, falling in $9(24.3 \%)$ cases, and falling from height in $3(8.2 \%)$ down cases. According to the Denis-Weber classification, $31(82.8 \%)$ cases were classified as type B and $6(16.2 \%)$ cases were classified as type C. Tibiofibular incongruence was determined in $25(67.6 \%)$ cases in the external rotation test after the fixation of both malleolus. In all of these cases, syndesmosis repair was made by crossing 1 syndesmotic screw over the fibula plate. The medial malleolar fracture was fixed with a screw in 28 cases and with tension band technique in 9 cases. The preoperative TCS was measured as $6.05 \pm 1.86 \mathrm{~mm}$, postoperative TCS as $4.19 \pm 0.40 \mathrm{~mm}$, preoperative TFO as $5.03 \pm 2.93 \mathrm{~mm}$, postoperative TFO as $8.62 \pm 1.04$ $\mathrm{mm}$ and postoperative MCS as $4.11 \pm 0.57 \mathrm{~mm}$. Postoperative medial clear space of $5 \mathrm{~mm}$ and higher was determined in $7(18.9 \%)$ cases. The difference between preoperative and postoperative tibiofibular congruence measurement results was statistically significant (Table 1). The average union time of both malleolus was $7.73 \pm 1.61$ weeks.

There was no statistical relationship between the fracture type according to the Denis-Weber classification or preoperative syndesmosis dysfunction and postoperative medial clear space congruence $(p>$ 0.05) (Table 2).

\section{DISCUSSION}

The sequence of injury in supination-external rotation fractures is anterior tibiofibular ligament disruption (SER-I), oblique fibula fracture (SER-II), posterior tibiofibular ligament or posterior malleolar fracture (SER-III) followed by deltoid ligament rupture or medial malleolar fracture (SER-IV) [1]. In other words; according to Lauge-Hansen classification, if a bimalleolar fracture has occurred in SER type 4 injury, the deltoid ligament should be intact. But, there are studies suggesting that in bimalleolar fractures, a medial malleolar fracture may be concomitantly accompanied by deltoid ligament damage. However, these studies suggested that the deltoid ligament is not damaged in fractures of the supracollicular region. Even, this injury was associated with anterior collicular fractures [7, 8]. Bozscyk et al. [9] suggested that many fibular fractures traditionally recognized as supination injuries actually can be caused by a pronation mechanism. They stated that the combination of a posterior malleolar fracture and an anterior colliculus fracture of the medial malleolus is indicative of the pronation mechanism [9]. In our study, there were radiographic deltoid insufficiency findings in $18.9 \%$ of the bimalleolar fractures with supracollicular medial malleolar fractures. Then, SER

\section{Table 2. Relationship between preoperative syndesmosis dysfunction and Denis-Weber classification and postoperative medial clear space}

\begin{tabular}{lccc}
\hline & \multicolumn{2}{c}{ Status of preoperative syndesmotic joint } & $p$ value \\
\hline Postoperative medial clear space & Impaired $(\mathbf{n}=\mathbf{2 5})$ & Intact $(\mathbf{n}=\mathbf{1 2})$ \\
& $4.12 \pm 0.67$ & $4.08 \pm 0.29$ & 0.857 \\
Postoperative medial clear space & \multicolumn{2}{c}{ Denis-Weber } \\
\hline
\end{tabular}

Data are shown as mean \pm standard deviation. 
type bimalleolar fractures with anterior colliculus fractures in the literature may be a result of the inability of the Lague-Hansen classification system to describe the mechanism of fracture. On the other hand, bimalleolar fractures, even though large medial malleolar fragment fracture, may be accompanied by deltoid ligament damage.

Lauge-Hansen proposed a fracture classification system that is commonly used to evaluate ankle fractures. Based on early experiments on manually created fractures in cadaver ankles, Lauge-Hansen reported that characteristic fracture models appear repeatedly depending on both the position of ankle and the direction of deforming force [11]. The critical review of the study by Lauge-Hansen revealed the lack of a controlled deforming force (since it is applied manually), the lack of information on the position of ankle (such as plantar flexion versus neutral position or dorsiflexion versus neutral position) and uncertainties regarding additional lateral bearing during the test [12]. The deep deltoid component is the strongest part of the ligament and functions as a resistance against external rotation of the ankle when the foot in dorsiflexion [7]. We think that the emergence of findings in our study may be a result of the inability of the Lauge-Hansen system to describe the position of the foot in sagital plane (flexion, neutral or extension). For example, the deep deltoid ligament is expected to be stretched with an ankle in dorsiflexion. The external rotation (ER) force applied to the talus may also damage to the deltoid ligament. If the force applied by the continuing talus rotation to the ankle mortise is forcible, it may contribute to the mechanism of medial malleolar fracture.

Tornetta [7] found that $26 \%$ of cases with bimalleolar fracture had deltoid insufficiency based on the evaluation in the peroperative stress test. And, this was associated with a small medial malleolar fragment fracture, and it was suggested that supracollicular fractures had no deltoid ligament injury [7]. Fukuyama et al. [8] evaluated the deltoid damage using MR images based on the same study. They reported that the deltoid ligament was injured in $50 \%$ of cases with SER bimalleolar fractures, and there was a relationship between small fragments associated with anterior colliculus and deltoid ligament injury. There was no difference in the size of fragment between the medial malleolar fractures with fully ruptured deltoid and those with intact deltoid [8]. However, in this study, the evaluation was made only based on MR findings. It gave no information about instability findings in stress radiographs. We believe that the fact that deltoid ligament damage was less commonly seen in our study might be caused by the inclusion of only supracollicular fractures in the study and/or the ability to display soft tissue structures that did not show radiologic instability finding in MR.

In our clinic, the instability check has been routinely performed with external rotation test under fluoroscopy during peroperative evaluation. Although the deltoid ligament was not intervened in any way during the peroperative period, $18.9 \%$ of the cases had a high MCS in the postoperative period. We think that this is associated with the insufficiency of the Lauge-Hansen classification system to describe medial ligament injury. Since when a surgeon who relies on the LaugeHansen classification evaluates the peroperative stress radiography after fixing the bimalleolar fracture, the MCS should be routinely normal in this patient according to the Lauge-Hansen classification, hence this might be the result of the fact that he/she failed to evaluate the MCS or to pay attention as required when evaluating the MCS.

Manual external rotation or gravity stress tests are considered as gold standard in the radiographic diagnosis of ankle instability [13]. In Stage 4 injuries, some of cases with medial injuries that do not require surgical treatment may be diagnosed with instability in external rotation or gravity stress tests [14]. Some recent studies reported that weight-bearing radiograph is a physiologic test of ankle stability, simulating the loads which the patient will put on the joint in the joint and ligament recovery period $[14,15]$. We also used weight-bearing radiographs in our patients. They are still gold standard stress tests in decision-making for ankle instability. This may be considered as one of the limitations of our study. On the other hand, in the case of abnormal medial clear spaces that can be detected even in weight-bearing radiographs, these may be expected to increase if stress tests are performed. Therefore, we believe that our study could provide sufficient evidence on radiographic findings of deltoid ligament injury which may be associated with SER-4 bimalleolar fractures.

The fact that the cases with high MCS were not validated by MR images may be considered as a lim- 
itation of our study. Because there may be no correlation between stress radiographs and soft tissue injuries detected by MR imaging $[5,13]$. On the other hand, MR may probably cause overestimation of complete ligament ruptures in cases with ankle fracture [4]. Also, magnetic resonance imaging may be not an ideal test for acute ankle fractures. Firstly, there is no clinical study suggesting that MRI includes diagnostic test performance statistics (sensitivity, specificity) to detect the degree of deltoid ligament injury [14].

Radiographic evaluation of MCS abnormal increase is an indirect method ssed in defining deltoid ligament injury [2]. Therefore, MCS alone may be insufficient to define deltoid ligament injury [13, 16, 17]. In our study, no deltoid ligament repair was performed in any case we detected an increase in medial clear space during the follow-up period. We also did not have records of functional and pain scores.

\section{Limitations}

Limitation of our study is that the correlation between MCS values we detected and deltoid ligament injury could not be demonstrated with more specific methods and the effect of this condition on clinical and functional outcomes was unknown. Other limitations of our study may include its retrospective nature, the low number of cases, and the lack of our knowledge on functional results. Because proving the clinical reflection of the significant radiologic findings obtained will add value to the study. This can be revealed only by a prospective, randomize-controlled study that has a long-term follow-up and a sufficient number of cases, and includes functional and clinical symptoms.

\section{CONCLUSION}

In SER type 4 bimalleolar fractures with a large medial malleolar fragment fracture, weight-bearing radiographs may show an increase in MCS. This means that a medial malleolar fracture in bimalleolar fractures may be also accompanied by deltoid ligament injury. The Lauge-Hansen classification system may be insufficient to identify a medial ligament injury. In SER Type 4 bimalleolar fractures, peroperative MCS should be evaluated, and the presence of instability findings should be warning to the surgeon in terms of deltoid ligament injury.

\section{Authors' Contribution}

Study Conception: AY, MY, TOB, BK, Yİ, SSD, MÇK, HG; Study Design: AY, MY, TOB, BK, Yİ, SSD, MÇK, HG; Supervision: AY, MY, TOB, BK, Yİ, SSD, MÇK, HG; Funding: AY, MY, TOB, BK; Materials: AY, MY, TOB, BK; Data Collection and/or Processing: AY, MY, Yİ, SSD, MÇK; Statistical Analysis and/or Data Interpretation: AY, MY, TOB, BK, Yİ, SSD, MÇK, HG; Literature Review: AY, MY, TOB, BK, Yİ, SSD, MÇK, HG; Manuscript Preparation: AY, MY, TOB, BK, Yİ, SSD, MÇK, HG and Critical Review: AY, MY, TOB, BK, Yİ, SSD, MÇK, HG.

\section{Conflict of interest}

The author disclosed no conflict of interest during the preparation or publication of this manuscript.

\section{Financing}

The author disclosed that they did not receive any grant during conduction or writing of this study.

\section{REFERENCES}

1. Kosuge DD, Mahadevan D, Chandrasenan J, Pugh H. Managing type II and type IV Lauge-Hansen supination external rotation ankle fractures: current orthopaedic practice. Ann R Coll Surg Engl 2010;92:689-92.

2. DeAngelis NA, Eskander MS, French BG. Does medial tenderness predict deep deltoid ligament incompetence in supination-external rotation type ankle fractures? J Orthop Trauma 2007;21:244-7.

3. Tartaglione JP, Rosenbaum AJ, Abousayed M, DiPreta JA. Classifications in brief: Lauge-Hansen classification of ankle fractures. Clin Orthop Relat Res 2015;473:3323-8.

4. Warner SJ, Garner MR, Hinds RM, Helfet DL, Lorich DG. Correlation between the Lauge-Hansen classification and ligament injuries in ankle fractures. J Orthop Trauma 2015;29:5748.

5. Cabuk H, Celebi F, İmren Y, Dedeoğlu SS, Kır MC, Uyanık AF et al. Compatibility of Lauge-Hansen classification between plain radiographs and magnetic resonance imaging in ankle fractures. J Foot Ankle Surg 2018;57:712-5.

6. Gardner MJ, Demetrakopoulos D, Briggs SM, Helfet DL, Lorich DG. The Ability of the Lauge-Hansen classification to predict ligament injury and mechanism in ankle fractures: an MRI study. J Orthop Trauma 2006;20:267-72.

7. Tornetta PIII. Competence of the deltoid ligament in bimalleolar ankle fractures after medial malleolar fixation. J Bone Joint Surg Am 2000;82:843-8.

8. Fukuyama JM, Pires RES, Labronici PJ, Hungria JOS, Decusati RL. Bimalleolar ankle fracture: a simple fracture? Acta Ortop Bras 2017;25:48-51. 
9. Boszczyk A, Fudalej M, Kwapisz S, Błonski M, Kicinski M, Kordasiewicz B et al. X-ray features to predict ankle fracture mechanism. Forensic Sci Int 2018;291:185-92.

10. Warner SJ, Garner MR, Hinds RM, Helfet DL, Lorich DG. Correlation between the Lauge-Hansen classification and ligament injuries in ankle fractures. J Orthop Trauma 2015;29:5748.

11. Gardner MJ, Demetrakopoulos D, Briggs SM, Helfet DL, Lorich, DG. Malreduction of the tibiofibular syndesmosis in ankle fractures. Foot Ankle Int 2006;27:788-92.

12. Budny AM, Young BA. Analysis of radiographic classifications for rotational ankle fractures. Clin Podiatr Med Surg 2008;25:139-52.

13. van den Bekerom MPJ, Mutsaerts ELAR, van Dijk CN. Evaluation of the integrity of the deltoid ligament in supination ex- ternal rotation ankle fractures: a systematic review of the literature. Arch Orthop Trauma Surg 2009;129:227-35.

14. Hoshino CM, Nomoto EK, Norheim EP, Harris TG. Correlation of weightbearing radiographs and stability of stress positive ankle fractures. Foot Ankle Int 2012;33:92-8.

15. Weber M, Burmeister H, Flueckiger G, Krause FG. The use of weightbearing radiographs to assess the stability of supination-external rotation fractures of the ankle. Arch Orthop Trauma Surg 2010;130:693-8.

16. Gougoulias N, Khanna A, Sakellariou A, Maffulli N. Supination-external rotation ankle fractures. Clin Orthop Relat Res 2010;468:243-51.

17. Krintensen KD, Hansen T. Closed treatment of ankle fractures: stage II fractures followed twenty years. Acta Orthop Scand 1985;56:107-9. 